\title{
Chemogenetic modulation of histaminergic neurons in the tuberomammillary nucleus alters territorial aggression and wakefulness
}

\section{Fumito Naganuma}

Tohoku Medical and Pharmaceutical University

Tadaho Nakamura ( $\nabla$ tadahonakamura@tohoku-mpu.ac.jp )

Tohoku Medical and Pharmaceutical University

Hiroshi Kuroyanagi

Tohoku Medical and Pharmaceutical University

Masato Tanaka

Tohoku Medical and Pharmaceutical University

\section{Takeo Yoshikawa}

Tohoku University

Kazuhiko Yanai

Tohoku University

Nobuyuki Okamura

Tohoku University

\section{Research Article}

Keywords: Histaminergic neuron, tuberomammillary nucleus, locomotor activity, aggressive behaviour, sleep-wake cycle, DREADDs

Posted Date: April 20th, 2021

DOI: https://doi.org/10.21203/rs.3.rs-414232/v1

License: (a) (1) This work is licensed under a Creative Commons Attribution 4.0 International License. Read Full License 


\section{Abstract}

Designer receptor activated by designer drugs (DREADDs) techniques are widely used to modulate the activities of specific neuronal populations during behavioural tasks. However, DREADDs-induced modulation of histaminergic neurons in the tuberomammillary nucleus ( $\mathrm{HA}^{\mathrm{TMN}}$ neurons) has produced inconsistent effects on the sleep-wake cycle, possibly due to the use of Hdc-Cre mice driving Cre recombinase and DREADDs activity outside the targeted region. Moreover, previous DREADDs studies have not examined locomotor activity and aggressive behaviours, which are also regulated by brain histamine levels. In the present study, we investigated the effects of $\mathrm{HA}^{\mathrm{TMN}}$ activation and inhibition on the locomotor activity, aggressive behaviours and sleep-wake cycle of Hdc-Cre mice with minimal nontarget expression of Cre-recombinase. Chemoactivation of $\mathrm{HA}^{\mathrm{TMN}}$ moderately enhanced locomotor activity in a novel open field. Activation of $\mathrm{HA}^{\mathrm{TMN}}$ neurons significantly enhanced aggressive behaviour in the resident-intruder test. Wakefulness was increased and non-rapid eye movement (NREM) sleep decreased for an hour by $\mathrm{HA}^{\mathrm{TMN}}$ chemoactivation. Conversely $H \mathrm{~A}^{\mathrm{TMN}}$ chemoinhibition decreased wakefulness and increased NREM sleep for 6 hours. These changes in wakefulness induced by $H A^{T M N}$ modulation were related to vigilance status transition. These results indicate the influences of $\mathrm{HA}^{\mathrm{TMN}}$ neurons on exploratory activity, territorial aggression, and wake maintenance.

\section{Highlights}

- DREADDs was used to modulate TMN histaminergic ( $\mathrm{HA}^{\mathrm{TMN}}$ ) neuron activity in mice

- Excitation enhanced novel locomotor activity but reduced non-novel locomotor activity

- Activation of $H A^{T M N}$ neurons also promoted aggression against intruders

- Modulation altered wakefulness through regulation of sleep-wake transitions

- $H A^{T M N}$ neurons project to regions regulating locomotion, aggression and wakefulness

\section{Introduction}

Histaminergic neurons in the tuberomammillary nucleus $(T M N)$ of the posterior hypothalamus ( $\mathrm{HA}^{\mathrm{TMN}}$ neurons) modulate a multitude of physiological processes and behaviours, and altered neuronal histamine signalling is associated with changes in the sleep-wake cycle, motor activity and aggression among other behavioural effects ${ }^{1,2}$. Several technical challenges have limited research on the specific behavioural functions of $\mathrm{HA}^{\mathrm{TMN}}$ neurons. Notably, histamine $(\mathrm{HA})$ receptors are widely distributed throughout the central nervous system (CNS) and peripheral tissues, limiting the utility of pharmacologic modulation, while clusters of HA neurons may be difficult to stimulate specifically using implanted electrodes. Alternatively, chemogenetic and optogenetic techniques allow for the modulation of neuronal activities with regional, cellular and temporal specificity ${ }^{3,4}$. However, such techniques applied to HA ${ }^{\mathrm{TMN}}$ neurons have also yielded inconsistent effects on the sleep-wake cycle. In one recent study, chemogenetic inhibition decreased wakefulness and increased non-rapid eye movement (NREM) sleep 
without changing REM sleep during active periods (Yu et al., 2019). Similarly, another optogenetic study reported that acute silencing of $H A^{T M N}$ neurons during wakefulness promoted NREM sleep, but not REM sleep (Fujita et al., 2017). However, Venner et al. (2019) reported that optogenetic inhibition of HATMN neurons during wakefulness did not alter the duration of NREM sleep. Furthermore, chemogenetic activation of $\mathrm{HA}^{\mathrm{TMN}}$ neurons at Zeitgeber time (ZT) 3 did not alter the sleep/wake ratio or electroencephalogram (EEG) spectra ${ }^{5}$.

In addition to the sleep-wake cycle, the brain histaminergic system is involved in the regulation of locomotor activity and aggressive behaviours. Genetic deletion (knockout, KO) of histamine $\mathrm{N}$ methyltransferase (Hnmt) (EC 2.1.1.8), which metabolizes histamine into inactive 1-methylhistamine, induced a sustained elevation of extracellular histamine concentration in the mouse CNS and concomitantly reduced locomotor activity during the dark periods, enhanced aggressive behaviours and disrupted the normal sleep-wake cycle without changing the total amount of wakefulness ${ }^{6}$.

We speculated that these discrepancies in the behavioural effects of $\mathrm{HA}^{\mathrm{TMN}}$ neuron modulation could reflect relatively minor differences in behavioural assessment methods and the emotional status of the animal. Furthermore, we suggest that the histidine decarboxylase (Hdc) (EC 4.1.1.22)-Cre mouse line may provide more cell type-specific chemogenetic or optogenetic manipulation of $\mathrm{HA}^{\mathrm{TMN}}$ neuron activity ${ }^{2,7}$. Therefore, we re-examined histaminergic system functions in regulation of the sleep-wake cycle, locomotor activity and aggressive behaviours using designer receptor exclusively activated by designer drugs (DREADDs) in Hdc-Cre mice, and further examined the projection distribution of $H A^{T M N}$ fibres by immunohistology to identify the neural circuits underlying the behavioural effects of $H A^{T M N}$ neuron activity.

\section{Results}

\subsection{Chemogenetic receptor expression by $H A^{T M N}$ neurons after microinjection}

Cell type-specific expression of chemogenetic receptors is crucial for establishing relationships between activity changes and behaviour. We therefore first confirmed that expression of chemogenetic receptors was exclusive to $\mathrm{HA}^{\mathrm{TMN}}$ neurons in the posterior hypothalamus by scanning brain slices obtained after behavioural experiments for immunoreactivity to the vector marker mCherry. Indeed, mCherry immunoreactivity (brown) was detected only in posterior hypothalamus (TMN) in mice injected with hM3Dq (Fig. 1A) or hM4Di (Fig. 1B). Moreover, nuclear c-Fos expression (black) was markedly higher in hM3Dq-mCherry-immunoreactive cells (Fig. 1A') compared to hM4Di-mCherry-immunoreactive cells (Fig. 1B') $3.0 \mathrm{~h}$ after administration of clozapine $\mathrm{N}$-oxide (CNO) $(0.3 \mathrm{mg} / \mathrm{kg}$ i.p.), consistent with greater neuronal activity specifically in CNO responsive cells ${ }^{8}$.

\subsection{Activation of $H A^{T M N}$ neurons enhanced locomotor activity in a novel environment}


Previous studies have yielded inconsistent or equivocal results regarding the effects of $\mathrm{HA}^{\mathrm{TMN}}$ neuron excitation or inhibition on mouse locomotor activity. Acute pharmacological inhibition of histamine decarboxylase (HDC) or deletion of the Hdc gene, interventions that would reduce brain histamine levels, have been reported to decrease locomotor activity in rodents ${ }^{9-13}$. However, a chronic increase in histamine level also decreased locomotor activity in dark periods, while an acute moderate increase in brain histamine was found to enhance locomotor activity 6,14,15. Histamine receptor stimulation/inhibition outside the TMN, effects of histamine on other neuronal populations and (or) compensatory changes after knockout may obscure effects of $H A^{T M N}$ neurons on locomotion. Therefore, we established a DREADDs system to assess the effects of direct acute activation or inhibition of $\mathrm{HA}^{\mathrm{TMN}}$ neurons. Chemogenetic activation of $H A^{T M N}$ neurons with CNO did not significantly alter locomotor activity during the first 30-min trial compared to saline (SA) (Fig. 2A-D), but there were significant differences in movement time and time in the central area between trials 1 and 2 (Fig. 2C and D). Similarly, inhibition of $\mathrm{HA}^{\mathrm{TMN}}$ neurons by hM4Di-CNO did not decrease mean locomotor activity during two 30-min trials compared to controls (Fig. 2E-H) but there were significant differences in total movement distance, average speed and total movement time between trials 1 and 2 (Fig. 2E-G). In the subgroup analyses, the hM3Dq-CNO group demonstrated increased total movement distance and average speed in the first trial (Fig. S1A and B). Time-resolved analyses indicated that these indices were significantly increased in the first 5-min period (Fig. S1A and B). In the second trial, however, hM3Dq-CNO significantly decreased movement distance and average speed (Fig. S1I and J). On the other hand, subgroup analysis of hM4Di did not reveal any significant differences (Fig. S1E-H and M-P). These results indicate that $\mathrm{HA}^{\mathrm{TMN}}$ neuron activation enhances locomotor activity in a novel environment but actually decreases activity in a non-novel environment, suggesting that HA signalling influences exploratory behaviour.

\subsection{Chemoactivation of $H A^{T M N}$ neurons increases territorial aggression}

Like locomotor activity, histaminergic influences on aggressive behaviours have been inconsistent across studies using pharmacologic manipulations or Hnmt KO ${ }^{6,16-18}$. Specific activation of $\mathrm{HA}^{\mathrm{TMN}}$ neurons by hM3Dq-CNO significantly increased the number and duration of aggressive behaviours (Fig. $3 A$ and $B$ ) compared to saline control in the resident-intruder test, and also tended to reduce the latency to first aggressive behaviour, although the difference did not reach statistical significance (Fig. 3C). Conversely, inhibition of $\mathrm{HA}^{\mathrm{TMN}}$ neurons by hM4Di-CNO had no significant influence on aggression compared to saline control (Fig. $3 \mathrm{~A}-\mathrm{C})$. These results indicate that the activation of $\mathrm{HA}^{\mathrm{TMN}}$ neurons triggers and maintains territorial aggression in mice.

\subsection{Modulation of $H A^{T M N}$ neuron activity alters the sleep-wake cycle}

Next, we examined whether specific activation of $H A^{T M N}$ alters the sleep-wake cycle by administering CNO or SA to AAV-hM3Dq mice at ZT3 (light period) or ZT12 (dark period). In two-arm crossover experiments, hM3Dq-CNO did not alter wake, NREM sleep, or REM sleep times within the first 6-h during light (Fig. 4A) or dark periods (Fig. S2A). Time-resolved analyses, however, revealed that CNO injection at 
ZT3 significantly increased total waking time and decreased NREM sleep time within the first $1 \mathrm{~h}$ (ZT3ZT4) while REM sleep time was not changed (Fig. 4B). The latency to the first NREM sleep episode was significantly extended by hM3Dq-CNO (Fig. 4E), while the number and the mean duration of waking, NREM sleep and REM sleep episodes during the first $1 \mathrm{~h}$ were not changed (Fig. $4 \mathrm{C}$ and $\mathrm{D}$, and table S1). The frequency of Wake to Wake transitions ( $W \forall W$ ) was significantly increased whereas the frequency of NREM to NREM transitions (NR $\forall$ NR) was significantly decreased in the hM3Dq-CNO group, indicating that activation of $H A^{T M N}$ neurons contributes to arousal maintenance (Fig. 4F). There were no differences in EEG power spectra during wakefulness, NREM sleep and REM sleep between CNO and SA groups (Fig. S4A). In addition, CNO injection at ZT12 did not alter sleep-wake status in sub-analyses (Fig. S2 and S4B, and Table S1).

We also examined whether specific inhibition of $\mathrm{HA}^{\mathrm{TMN}}$ neurons suppressed wakefulness by comparing AAV-hM4Di mouse responses to CNO and SA administered at ZT3 or ZT12. Injection of CNO decreased waking time and increased NREM sleep time during the dark periods (Fig. 5A), while REM sleep in the dark periods and all sleep and wake stages in the light periods did not differ between CNO and SA groups (Fig. 5A and S3). Analysis of sleep-wake status during the first $1 \mathrm{~h}$ after CNO injection revealed decreased waking duration and increased NREM sleep duration but no changes in REM sleep duration (Fig. 5B). The mean duration of NREM sleep was significantly increased by hM4Di-CNO at ZT12 (Fig. 5D and table S2), while the latencies to first NREM sleep and REM sleep episodes, mean durations of wakefulness and REM sleep and the number of individual sleep or waking episodes were not significantly changed (Fig. 5C-E). The number of W-W transitions was reduced while the number of NR-NR transitions was increased by hM4Di-CNO at ZT12 (Fig. 5F). Collectively, these findings demonstrate the HA ${ }^{T M N}$ neuron inhibition during the dark period impedes arousal maintenance. EEG power densities for each episode did not differ between NCO and SA groups (Fig. S4D). Further, CNO injection at ZT3 did not significantly alter sleep-wake status (Fig. S3 and S4C, Table S2). These results indicate that HA ${ }^{\text {TMN }}$ neuron activity during the dark period is crucial for maintaining arousal status.

\section{5. $\mathrm{HA}^{\mathrm{TMN}}$ neurons projects to brain regions known to regulate aggression, vigilance and the sleep-wake cycle}

We then investigated the potential circuits mediating these effects of $\mathrm{HA}^{\mathrm{TMN}}$ neuron chemoactivation/chemoinhibition on locomotion, aggression and wakefulness by conducting serial mCherry immunostaining to identify projection targets. Although the brains were harvested 2 months after AAV microinjection, mCherry-immunoreactive somata were found exclusively in the ventral and caudal TMN (Fig. 6A and B). In contrast, mCherry-positive histaminergic fibres were observed in multiple brain regions at 6 months post-AAV microinjection, including the bed nucleus of the stria terminalis (BNST) and substantia innominata (SI) regions of the preoptic area (Fig. 6C), wide regions of hypothalamus but particularly in the lateral hypothalamus (LH) (Fig. 6D), central amygdala (CeA) (Fig. 6E) and periaqueductal grey (PAG), especially the ventrolateral PAG (vIPAG) (Fig. 6F). These regions may mediate the effects of $\mathrm{HA}^{\mathrm{TMN}}$ neuron activity on exploratory locomotion, territorial aggression and the sleep-wake cycle. 


\section{Discussion}

In the present study, we demonstrate that the specific modulation of $\mathrm{HA}^{\mathrm{TMN}}$ neuron activity by DREADDs can influence exploratory locomotion, territorial aggression and the sleep-wake cycle of mice. Although specific activation of $\mathrm{HA}^{\mathrm{TMN}}$ neurons did not significantly alter overall locomotion in the two-arm crossover trial, activation moderately increased locomotion in trial 1 (first exposure) and moderately decreased locomotion in trial 2. In contrast to these modest effects on locomotion, activation of $\mathrm{HA}^{\mathrm{TMN}}$ neurons markedly enhanced aggression of male mice toward an intruder male. Furthermore, $\mathrm{HA}^{\mathrm{TMN}}$ neuron activation increased wakefulness for an hour in the light periods, whereas inhibition significantly decreased wakefulness during the dark periods. Transitions in sleep-wake status were also regulated by $H A^{T M N}$ neuron activity. Finally, we show that TMN histaminergic neurons project to brain regions implicated in fear, aggression and arousal, such as the BNST, LH, CeA and vIPAG.

The $H A^{T M N}$ neurons of rodents are distributed in five clusters, E1, E2 and E3 in the ventral area, E4 in the medial dorsal area and E5 more diffusely in the dorsal area ${ }^{19}$. At 2-3 months after injection of HdchM3Dq or Hdc-hM4Di vector, mCherry-positive histaminergic neurons were localised primarily in lateral E1 and E2 (Fig. 1A and B), a distribution comparable to that of Hdc-Cre mice crossed with a Cre-dependent tdTomato reporter line ${ }^{7}$. In addition, however, immunostaining of brain Sect. 6 months after microinjection revealed additional mCherry-positive histaminergic neurons in E3 and E5 (Fig. 6A and B). A transgene downstream of the human synapsin gene (hSyn) promoter in the AAV vector was exclusively expressed in neurons, and AAV8 induced long-term upregulation of the transgene ${ }^{20,21}$. Therefore, a wider distribution of immunoreactive histaminergic neurons in the present study may result not only from a longer time period for expression but also from use of the hdc promoter. While we assume that the observed behavioural effects are due mainly to the activities of E1 and E2 histaminergic neurons (Fig. 1), contributions of E3 and E5 neurons cannot be totally excluded.

Previous studies have clearly demonstrated that $\mathrm{HA}^{T M N}$ neurons project to broad regions of the brain, including BSNT, LH, CeA and vIPAG ${ }^{22,23}$. Our projection study confirmed these findings and revealed a particularly dense projection to the bilateral vIPAG (Fig. 6F). Neurons of the vIPAG are involved in the regulation of locomotor activity ${ }^{24}$, aggressive behaviours ${ }^{25}$ and the sleep-wake cycle ${ }^{26-28}$, consistent with our behavioural results. Antegrade and retrograde optogenetics experiments are warranted to elucidate the precise circuit mechanisms.

A positive association between hypothalamic histamine release and physiological locomotor activity was first reported in $1992^{29}$ and thereafter evidence has accrued for important contributions of HA ${ }^{T M N}$ neuronal excitation to locomotor activity, mainly based on experiments in which histamine production or $H_{1}$ and $H_{3}$ receptors were inhibited ${ }^{9,12,30,31}$. In these loss-of-function studies, locomotor activity was generally reduced by inhibition of histaminergic signalling in the CNS, but gain-of-function studies in which brain histamine levels were increased have yielded unexpected and inconsistent results $6,14,15$. We found no significant difference in locomotion between CNO and control groups in the two-arm crossover 
experiment (Fig. 2), even in subgroup intracohort analysis between trials (data not shown). However, we found that $H A^{T M N}$ activation increased locomotor activity in the Hdc-hM3Dq intercohort analysis for trial 1 (Fig. S1) and decreased activity in trial 2, suggesting that $H A^{T M N}$ neuron activation enhances locomotor activity in a novel environment ${ }^{30,32}$. Increased locomotor activity by specific $\mathrm{HA}^{\mathrm{TMN}}$ chemogenetic activation was also reported by $\mathrm{Yu}$ et al. ${ }^{33}$ but they observed a greater and more sustained increase, possibly due to administration of $5 \mathrm{mg} / \mathrm{kg} \mathrm{CNO}$, which may induce nonspecific neuronal activation ${ }^{34}$. On the other hand, $H A^{T M N}$ neuron activation decreased locomotor activity in trial 2 (Fig. S1). Histaminergic activation also contributes to memory retrieval ${ }^{35-37}$, so this reduced locomotor activity in trial 2 may be due to strong contextual memory for an otherwise anodyne environment, resulting in lower exploratory motivation. In contrast, $\mathrm{HA}^{\mathrm{TMN}}$ suppression by hM4Di-CNO did not significantly alter locomotor activity (Fig. 2 and S1). However, chemogenetic inhibition of HA ${ }^{T M N}$ by $1 \mathrm{mg} / \mathrm{kg}$ CNO injection significantly decreased locomotion in dark periods ${ }^{38}$. We performed open-field tests with a much lower CNO dose during the light periods, the resting phase for nocturnal animals, so further studies are needed to evaluate effects of loss-of-function on locomotor activity during the dark periods.

In contrast to the subtle effects on locomotion, $\mathrm{HA}^{\mathrm{TMN}}$ neuron activation clearly enhanced territorial aggression (Fig. 3). Previous studies have suggested that elevated brain histamine levels induce aggression via $\mathrm{H}_{2}$ receptor activation ${ }^{6,16,17}$, consistent with the current results. We speculate that two mechanisms underlie increased aggression upon $\mathrm{HA}^{\mathrm{TMN}}$ neuron activation, direct modulation of neural pathways mediating aggression and modulation via effects on the circadian rhythm. One potential pathway is the ventromedial hypothalamus, ventrolateral subdivision (VMHvl)-vIPAG circuit ${ }^{39}$, as the $\mathrm{VMHvl}$ is innervated by the $H A^{T M N}$ and induces aggression upon stimulation ${ }^{23,40}$. The $H A^{T M N}$ also innervates the vIPAG (Fig. 6), which transforms higher level neuronal signals into aggressive actionspecific codes ${ }^{39}$. A second possible pathway is the suprachiasmatic nucleus ( $\mathrm{SCN}$ )-subparaventricular zone (SPZ)-VMHvl circuit. The SCN is the master circadian pacemaker in the mammalian brain, and sends dense axonal outputs to the SPZ, which negatively regulates aggression via the $\mathrm{VMHvl}{ }^{41}$. The $H A^{T M N}$ also projects to the SCN ${ }^{23,42}$ and has an inhibitory effect on $S C N$ neuronal activity through $\mathrm{H}_{1}$ and $\mathrm{H}_{2}$ receptor activation ${ }^{43-45}$. Additional projection mapping and chemogenetic activation studies are required to clarify the contributions of these pathways to territorial aggression induced by $\mathrm{HA}^{\mathrm{TMN}}$ neuron excitation.

To our knowledge, only three previous studies have used Hdc-Cre mice to examine the functions of $\mathrm{HA}^{\mathrm{TMN}}$ neurons in regulation of the sleep-wake cycle ${ }^{5,7,38}$. Chemogenetic inhibition of $\mathrm{HA}^{\mathrm{TMN}}$ neurons during the dark periods decreased wakefulness and increased NREM sleep duration in a different Hdc-Cre mouse line, and these responses were accompanied by increased EEG $\delta$ power and decreased EEG power at higher frequencies $(8-30 \mathrm{~Hz})^{38}$. A study using the same Hdc-Cre line employ in the current study found that optogenetic inhibition of $H A^{T M N}$ neurons by archaerhodopsin (Arch) 3.0 during wakefulness in the dark periods promoted NREM sleep and increased the number of NREM episodes but not NREM duration, 
and had no effect on EEG spectra ${ }^{7}$. Although we found prolonged mean NREM duration without alteration in the number of episodes (Fig. $5 \mathrm{C}$ and D) and no differences in the EEG power spectra for wake and all sleep stages (Fig. S4), the inhibitory experiments in the present study revealed an important role for $\mathrm{HA}^{\mathrm{TMN}}$ neurons in maintaining wakefulness during the dark periods, in accord with the aforementioned studies. However, inhibition of $\mathrm{HA}^{\mathrm{TMN}}$ neurons with ArchT during wakefulness did not impact NREM sleep in experiments using another Hdc-Cre line ${ }^{5}$. These authors also induced chemogenic activation of $\mathrm{HA}^{\mathrm{TMN}}$ neurons by $\mathrm{CNO}$ injection at $\mathrm{ZT} 3$ and found no alterations in the relative durations of sleep and wake stages or EEG spectra ${ }^{5}$. In the present study, we also found no changes in the sleepwake cycle following $\mathrm{HA}^{\mathrm{TMN}}$ neuron activation in the overall analysis (Fig. 4A-C). In sub-analysis, however, Hdc-hM3Dq mice demonstrated increased wakefulness in the first hour after CNO injection at ZT3 (Fig. 4B). Venner et al. (2019) also found that a new environment augmented the increased wakefulness and reduced NREM observed in response to $H A^{T M N}$ neuron activation during the light periods. We suggest that the significant increase in wakefulness observed during the first hour may be influenced by the novel environment of the experimental condition.

Previous electrophysiological studies have identify some TMN neurons as 'Waking-on' due to predominant activation during wakefulness and complete silence during NREM and REM sleep ${ }^{46-48}$. These neurons also exhibited a pronounced delay in firing during transitions from sleep to wakefulness and a long delay to an arousing stimulus, indicating important contributions to the maintenance of wakefulness and vigilance, but not to state induction ${ }^{47}$. Our findings from transition analyses, which showed W-W promotion by $H A^{T M N}$ activation and reduction by inhibition provide further support for the role of $\mathrm{HA}^{\mathrm{TMN}}$ neurons in arousal maintenance (Fig. 4F and 5F). Steininger et al. (1999) also suggested that some histaminergic neurons in rats discharged at high rates during REM sleep, termed 'REM-related neurons'. However, we found no relation between $\mathrm{HA}^{\mathrm{TMN}}$ neuron activity and REM sleep.

As previously described, the Hdc-Cre mouse line used in the present study has high specificity with minimal Cre recombinase expression outside the TMN; however, it also exhibits only $\sim 50 \%$ penetrance ${ }^{7}$. Thus, at least half of the Hdc-neuronal population was not modulated by the chemogenetic approach, which may explain why we did not observe the significant changes in locomotor activity and REM sleep reported in previous studies using a different Cre recombinase line with higher penetrance ${ }^{5,38}$. Further, several studies suggest that $H A^{T M N}$ neurons are functionally heterogeneous ${ }^{7,49-51}$ and form distinct projections to influence unique physiological functions and behaviours. Further work is required to determine the individual $\mathrm{HA}^{\mathrm{TMN}}$ pathways responsible for the observed changes in locomotor activity, aggression and the sleep-wake cycle. Furthermore, we found no correlations between behavioural changes across groups (data not shown). Larger cohorts for greater statistical power and projectionspecific activation methods may be required to identify such relationships.

In conclusion, the specific modulation of $\mathrm{HA}^{\mathrm{TMN}}$ neuron activity altered exploratory locomotion, territorial aggression and wake maintenance in mice. These responses may be explained by projections from 
$H A^{T M N}$ neurons to the POA, CeA, LH and vIPAG.

\section{Materials And Methods}

\subsection{Animals}

All animal care and use protocols were conducted in accordance with the Standards of Humane Care and Use of Laboratory Animals of Tohoku Medical and Pharmaceutical University, Sendai, Japan. All experiments involving animals also complied with the Guidelines for the Proper Conduct of Animal Experiments of the Ministry of Education, Culture, Sports, Science and Technology, Japan, and the ARRIVE guidelines for reporting animal research ${ }^{52}$. All animal experiments including the use of genetically modified mice were approved by the Tohoku Medical and Pharmaceutical University Animal Experiment Committee (registration number: 19004-cn) and Tohoku Medical and Pharmaceutical University Centre for Gene Research (registration number: 2018-19). All efforts were made to reduce the number of animals used and minimize animal suffering.

For this study, we used a mutant mouse line expressing Cre recombinase under control of the Hdc

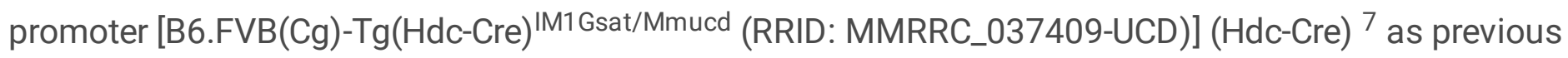
studies reported target specificity of $94.7 \%$ (albeit with penetrance of only $48.8 \%)^{7}$. Only male heterozygous Hdc-Cre mice were used for these experiments. All mice were group-housed in a temperature-controlled $\left(23^{\circ} \mathrm{C} \pm 1^{\circ} \mathrm{C}\right)$ and humidity-controlled $(55.0 \% \pm 5.0 \%)$ animal room under a $12: 12 \mathrm{~h}$ light:dark cycle [lights on at 8:00 (ZT0), lights off at 20:00 (ZT12)] with ad libitum access to standard rodent chow (CLEA Japan, Tokyo, Japan) and water.

\subsection{Genotyping}

Genotyping was performed in accordance with protocol MMRRC_032079-UCD. Briefly, genomic DNA was extracted from tail biopsies and analysed by polymerase chain reaction (PCR) using the KOD FX Neo enzyme (TOYOBO, Osaka, Japan). The PCR reaction protocol consisted of incubation at $94^{\circ} \mathrm{C}$ for 2 min, followed by 35 cycles of $98^{\circ} \mathrm{C}$ for $10 \mathrm{~s}, 60^{\circ} \mathrm{C}$ for $30 \mathrm{~s}$ and $68^{\circ} \mathrm{C}$ for $40 \mathrm{~s}$ under control of a T100 thermal cycler (Bio-Rad, Hercules, CA). The primer sequences were as follows: Hdc-Cre (575 bp): forward, 5'-AGC CTC CTC TGT CTG TCT GC; reverse, 5'-CCC CAG AAA TGC CAG ATT ACG TAT. WT (220 bp): forward, 5'CTC ACC CAA ATC CAA CGA CT; reverse, 5'-GCA GTT GCC TAG CAA CAC AC.

\subsection{Surgery}

Adult male Hdc-Cre mice (10-14 weeks old) were anaesthetised using a mixture of $0.3 \mathrm{mg} / \mathrm{kg}$ medetomidine (Zenoaq, Fukushima, Japan), $4 \mathrm{mg} / \mathrm{kg}$ midazolam (Astellas, Tokyo, Japan) and $5 \mathrm{mg} / \mathrm{kg}$ butorphanol (Meiji Seika Pharma, Tokyo, Japan), and fixed on a digital stereotactic instrument (940-XYZ, David Kopf, Tujunga, CA). A 100-nL volume of vector solution was microinjected into each TMN (AP - 2.4 $\mathrm{mm}, \mathrm{RL} \pm 1.0 \mathrm{~mm}$, DV - $5.1 \mathrm{~mm}$ from bregma). The vector solution contained either AAV8-hSyn-DIOhM3Dq-mCherry (titre: $2.2 \times 10^{13} \mathrm{vg} / \mathrm{mL}$, 44361-AAV8; Addgene, Watertown, MA) (AAV-hM3Dq) or AAV8DIO-hSyn-hM4Di-mCherry (titre: $2.5 \times 10^{13} \mathrm{vg} / \mathrm{mL}$, 4362-AAV8; Addgene) (AAV-hM4Di) to establish mouse 
models for chemogenetic activation and inhibition, respectively. Three weeks after microinjection, open field and resident-intruder tests were performed as described (Sect. 2.5). After the resident-intruder test, all mice were implanted with a head mount and electrodes (E363/76//NS/SPC, P1 Technologies, Manhattan Beach, CA) for EEG and electromyogram (EMG) recordings ${ }^{53}$. The EEG electrodes were inserted by threading through stainless steel screws while EMG electrodes were inserted directly into the neck extensor muscles.

\subsection{Histology}

After completion of behavioural studies, mice were deeply anaesthetised with a medetomidine/midazolam/butorphanol mixture and transcardially perfused with $15 \mathrm{~mL}$ phosphate buffered saline (PBS) followed by $50 \mathrm{~mL}$ of $10 \%$ formalin in PBS (Wako, Osaka, Japan). Mouse brains were harvested immediately and incubated in $10 \%$ formalin/PBS overnight at $4^{\circ} \mathrm{C}$, followed by incubation in $10 \%$ formalin/PBS with $20 \%$ sucrose at $4^{\circ} \mathrm{C}$ for 2 days. Brains were sliced into 3 series of $40-\mu \mathrm{m}$ coronal sections using a freezing cryostat (CM3050, LEICA, Wetzlar, Germany) and processed for immunohistochemistry.

For immunohistochemistry using diaminobenzidine (DAB) as the chromogen, sections were incubated with Fos antibody (1:10,000; SC-52; Santa Cruz Biotechnology, Dallas, TX) for two nights or ds-Red $(1: 10,000 ; Z 2496 \mathrm{~N}$; Clontech Laboratory, Mountain View, CA) overnight at room temperature, followed by incubation in the appropriate biotin-SP-conjugated secondary antibody $(1: 1,000$; Jackson ImmunoResearch, West Grove, PA) for $1 \mathrm{~h}$ at room temperature. Sections were incubated for $75 \mathrm{~min}$ in avidin-biotin-complex reagent (1:1000; Vectastain $A B C$ kit, Vector Lab, Burlingame, $C A$ ) at room temperature, washed and incubated in a solution of $0.06 \% \mathrm{DAB}$ (Wako) and $0.02 \% \mathrm{H}_{2} \mathrm{O}_{2}$ (Wako) for 2-5 min. Then, PBS containing $0.05 \% \mathrm{CoCl}_{2}$ (Wako) and $0.01 \% \mathrm{NiSO}_{4}\left(\mathrm{NH}_{4}\right)$ (Wako) was added to the DAB solution for staining (black).

Sections were mounted on Superfrost glass slides (Matsunami, Osaka, Japan), dehydrated, cleared and cover-slipped using Multi Mount 480 (Matsunami). The mounted brain sections were scanned using a BZ-X700 microscope (Keyence, Osaka, Japan) and composite images were generated by BZ-X Analyzer software (Keyence).

\subsection{Behavioural experiments}

All behavioural tests were performed with a two-arm crossover design by experimenters blinded to treatment history. Each microinjected mouse was examined first in the open-field test, followed by the resident-intruder test and sleep-wake cycle monitoring by EEG/EMG. Mice were randomly assigned to receive $\mathrm{CNO}$ or SA at the beginning of the first trial, then switched to the other drug for the second trial. Again, tests were analysed by experimenters blinded to mouse treatment history.

\subsubsection{Open-field test}

The open-field test was performed as previously described ${ }^{33}$. Briefly, mice were injected with CNO $(0.3$ $\mathrm{mg} / \mathrm{kg}$; Sigma, St Louis, MO) or saline vehicle (SA) as a control $30 \mathrm{~min}$ before the trial during the light 
period. Each mouse was placed into the centre of a $60 \mathrm{~cm} \times 60 \mathrm{~cm}$ square arena and allowed to explore freely for $30 \mathrm{~min}$. Total movement distance, average speed, total movement time and time spent in the central area were recorded and quantified using a video tracking system (EthoVision ${ }^{\circledR}$ XT, Ver. 13, Noldus, Wageningen, Netherlands) by an experimenter blinded to treatment history.

\subsubsection{Resident-intruder test}

The resident-intruder test was performed as previously described ${ }^{6}$. Briefly, resident mice were housed individually for 1 week before the testing day to increase territorial motivation. Then, the mouse was injected with CNO (0.3 mg/kg; Sigma) or SA 30 min before the trial during the light period. An unfamiliar male (intruder, C57BL6 WT, matched for age) was introduced into the resident cage and the two mice were allowed to interact freely for $10 \mathrm{~min}$. Aggression of the resident mouse was measured by the frequencies of 'mounting' (attempts to mount the intruder), 'chasing' and 'biting' (bites to dorsal/ventral regions of the intruder). The latency to first aggressive behaviour, number of aggressive behaviours and total duration of aggressive behaviours were measured. Another unfamiliar male mouse was used as an intruder in the second trial. All experimental data were video-recorded and analysed by an examiner blinded to treatment history.

\subsubsection{Sleep recordings and analysis}

A week after EEG/EMG electrode implantation, mice were connected to recording cables and habituated for 3 days in a cylinder chamber $(22 \mathrm{~cm}$ in diameter) placed within a soundproof electrically shielded box (SP-BOX/S, Shinfactory, Fukuoka, Japan) maintained at constant temperature $\left(23^{\circ} \mathrm{C} \pm 1^{\circ} \mathrm{C}\right)$ and humidity $(55.0 \% \pm 5.0 \%)$ under a 12:12h light-dark cycle (ZT0: 8:00, ZT12: 20:00) with food and water available ad libitum. The mice were injected with CNO (0.3 mg/kg; Sigma) or SA at 10:50 AM (10 min before ZT3) or 7:50 PM (10 min before ZT12), followed by EEG/EMG recording for $24 \mathrm{~h}$. Following an interval of at least 3 days, mice were switched to the other drug intervention group for another $24 \mathrm{~h}$ of EEG/EMG recording. The EEG/EMG signals were amplified (Biotex, Kyoto, Japan), digitised (AD16-16U/PIC/EV, CONTEC, Osaka, Japan) and recorded by a Vital Recorder system (Kissei Comtec, Nagano, Japan). Only the first 6 $\mathrm{h}$ of the $24 \mathrm{~h}$ period were analysed. Briefly, collected data were divided into 12-s epochs and scored manually for one of three sleep or wake states, wake, NREM sleep, or REM sleep, using SleepSign 3 (Kissei Comtec). Time spent in each state, episode frequency and episode duration were calculated in 1-h bins. Latencies to the first NREM and REM sleep episodes were measured as the period following wakefulness evoked by intraperitoneal (i.p.) injection. We also quantified sleep-wake status transitions for the first hour after CNO or SA injection. Sleep-wake status in two consecutive epochs was determined, then the frequencies of status transitions from the first epoch to the second epoch (Wake to Wake, Wake to NREM, NREM to NREM, NREM to Wake, NREM to REM, REM to REM and REM to Wake) were counted. The power spectral density of EEG signals in each stage was calculated with $0.25 \mathrm{~Hz}$ resolution by Fast Fourier Transform using SleepSign 3 software, then normalised for each animal by calculating the \% power of each bin relative to the total power from 0 to $20 \mathrm{~Hz}$.

\subsection{Statistical analysis}


All statistical analyses were performed using GraphPad Prism ${ }^{\circledR}$ version 8 (GraphPad Software, La Jolla, $\mathrm{CA}$ ). In behavioural experiments, the CNO group was compared to the SA group by two-way repeated measures ANOVA followed by Sidak's post hoc test or by Mann-Whitney $U$ test as indicated in the figure legends. All data are presented as the mean \pm standard error of the mean (S.E.M.) unless otherwise noted. Differences were considered significant at $P<0.05$. All statistical analysis was performed by experiments blinded to animal treatment history.

\section{Abbreviations}

AAV, adeno-associated virus; Arch, archaerhodopsin; BNST, bed nucleus of the stria terminalis; CeA, central amygdala; CNO, clozapine N-oxide; DAB, diaminobenzidine; DREADDs, Designer receptor activated by designer drugs; EEG, electroencephalogram; EMG, electromyogram; HA, histamine; Hdc, histidine decarboxylase; Hnmt, histamine $\mathrm{N}$-methyltransferase; LH, lateral hypothalamus; NREM, non-rapid eye movement; REM, rapid eye movement; SCN, suprachiasmatic nucleus; SI, substantia innominata; SPZ, subparaventricular zone; TMN, tuberomammillary nucleus; VMHvl, ventromedial hypothalamus, ventrolateral subdivision; vIPAG, ventrolateral periaqueductal grey; ZT, Zeitgeber time.

\section{Declarations}

\section{Data availability}

The datasets generated during and/or analysed during the current study are available from the corresponding author on reasonable request.

\section{Acknowledgements}

We thank the staff of the Animal Facility and Technical Service Division of Tohoku Medical and Pharmaceutical University for technical support.

\section{Funding}

This work was supported by a Japan Society for the Promotion of Science Grant-in-Aid for Scientific Research B (18H02771) to NO, Grant-in-Aid for Young Scientists (17K16753) and Grant-in-Aid for Scientific Research C (20K09204) to TN and a Grant-in-Aid for Young Scientists (19K16296) to FN.

\section{Competing interest}

The authors declare no competing interests.

\section{Author contributions}

FN: Conceptualisation, Methodology, Investigation, Writing-Original Draft, Visualisation, Funding acquisition. TN: Conceptualisation, Methodology, Validation, Resources, Formal analysis, Writing-Original 
Draft, Writing-Reviewing and Editing, Project administration, Funding acquisition. HK: Investigation, Formal analysis. MT: Investigation, Formal analysis. TY: Conceptualisation, Methodology, WritingOriginal Draft. KY: Writing- Reviewing and Editing, Supervision. NO: Conceptualisation, Writing-Reviewing and Editing, Supervision, Project administration, Funding acquisition.

\section{References}

1 Haas, H. L., Sergeeva, O. A. \& Selbach, O. Histamine in the nervous system. Physiological reviews 88, 1183-1241, doi:10.1152/physrev.00043.2007 (2008).

2 Yoshikawa, T., Nakamura, T. \& Yanai, K. Histaminergic neurons in the tuberomammillary nucleus as a control centre for wakefulness. British journal of pharmacology, doi:10.1111/bph.15220 (2020).

3 Deisseroth, K. Optogenetics: 10 years of microbial opsins in neuroscience. Nature neuroscience 18, 1213-1225, doi:10.1038/nn.4091 (2015).

4 Roth, B. L. DREADDs for Neuroscientists. Neuron 89, 683-694, doi:10.1016/j.neuron.2016.01.040 (2016).

5 Venner, A. et al. Reassessing the Role of Histaminergic Tuberomammillary Neurons in Arousal Control. The Journal of neuroscience : the official journal of the Society for Neuroscience 39, 8929-8939, doi:10.1523/jneurosci.1032-19.2019 (2019).

6 Naganuma, F. et al. Histamine N-methyltransferase regulates aggression and the sleep-wake cycle. Scientific reports 7, 15899, doi:10.1038/s41598-017-16019-8 (2017).

7 Fujita, A. et al. Hypothalamic Tuberomammillary Nucleus Neurons: Electrophysiological Diversity and Essential Role in Arousal Stability. The Journal of neuroscience : the official journal of the Society for Neuroscience 37, 9574-9592, doi:10.1523/jneurosci.0580-17.2017 (2017).

8 Naganuma, F. et al. Lateral hypothalamic neurotensin neurons promote arousal and hyperthermia. PLoS biology 17, e3000172, doi:10.1371/journal.pbio.3000172 (2019).

9 Dere, E. et al. Changes in motoric, exploratory and emotional behaviours and neuronal acetylcholine content and 5-HT turnover in histidine decarboxylase-KO mice. The European journal of neuroscience 20, 1051-1058, doi:10.1111/j.1460-9568.2004.03546.x (2004).

10 Kubota, Y. et al. Increased methamphetamine-induced locomotor activity and behavioral sensitization in histamine-deficient mice. Journal of neurochemistry 83, 837-845, doi:10.1046/j.14714159.2002.01189.x (2002).

11 Onodera, K., Yamatodani, A. \& Watanabe, T. Effects of alpha-fluoromethylhistidine on locomotor activity, brain histamine and catecholamine contents in rats. Methods and findings in experimental and clinical pharmacology 14, 97-105 (1992). 
12 Sakai, N., Onodera, K., Maeyama, K., Yanai, K. \& Watanabe, T. Effects of (S)-alpha fluoromethylhistidine and metoprine on locomotor activity and brain histamine content in mice. Life sciences 51, 397-405, doi:10.1016/0024-3205(92)90406-f (1992).

13 Sakai, N. et al. Effects of (S)-alpha-fluoromethylhistidine and (R)-alpha-methylhistamine on locomotion of W/Wv mice. Pharmacology, biochemistry, and behavior 46, 95-99, doi:10.1016/00913057(93)90323-I (1993).

14 Bristow, L. J. \& Bennett, G. W. Biphasic effects of intra-accumbens histamine administration on spontaneous motor activity in the rat; a role for central histamine receptors. British journal of pharmacology 95, 1292-1302, doi:10.1111/j.1476-5381.1988.tb11767.x (1988).

15 Ishizuka, T., Murakami, M. \& Yamatodani, A. Involvement of central histaminergic systems in modafinil-induced but not methylphenidate-induced increases in locomotor activity in rats. European journal of pharmacology 578, 209-215, doi:10.1016/j.ejphar.2007.09.009 (2008).

16 Nath, C., Gulati, A., Dhawan, K. N., Gupta, G. P. \& Bhargava, K. P. Evidence for central histaminergic mechanism in foot shock aggression. Psychopharmacology 76, 228-231, doi:10.1007/bf00432550 (1982).

17 Ray, A., Sharma, K. K. \& Sen, P. Effect of histaminergic drugs on footshock-induced aggressive behaviour in rats. European journal of pharmacology 73, 217-219, doi:10.1016/0014-2999(81)90094-7 (1981).

18 White, J. M. \& Rumbold, G. R. Behavioural effects of histamine and its antagonists: a review. Psychopharmacology 95, 1-14, doi:10.1007/bf00212757 (1988).

19 Inagaki, N. et al. An analysis of histaminergic efferents of the tuberomammillary nucleus to the medial preoptic area and inferior colliculus of the rat. Experimental brain research 80, 374-380, doi:10.1007/bf00228164 (1990).

20 Kügler, S., Kilic, E. \& Bähr, M. Human synapsin 1 gene promoter confers highly neuron-specific long-term transgene expression from an adenoviral vector in the adult rat brain depending on the transduced area. Gene therapy 10, 337-347, doi:10.1038/sj.gt.3301905 (2003).

21 Palomeque, J. et al. Efficiency of eight different AAV serotypes in transducing rat myocardium in vivo. Gene therapy 14, 989-997, doi:10.1038/sj.gt.3302895 (2007).

22 Inagaki, N. et al. Organization of histaminergic fibers in the rat brain. The Journal of comparative neurology 273, 283-300, doi:10.1002/cne.902730302 (1988).

23 Panula, P., Pirvola, U., Auvinen, S. \& Airaksinen, M. S. Histamine-immunoreactive nerve fibers in the rat brain. Neuroscience 28, 585-610, doi:10.1016/0306-4522(89)90007-9 (1989). 
24 Tovote, P. et al. Midbrain circuits for defensive behaviour. Nature 534, 206-212, doi:10.1038/nature17996 (2016).

25 Wright, K. M. \& McDannald, M. A. Ventrolateral periaqueductal gray neurons prioritize threat probability over fear output. eL ife 8, doi:10.7554/eLife.45013 (2019).

26 Hayashi, Y. et al. Cells of a common developmental origin regulate REM/non-REM sleep and wakefulness in mice. Science (New York, N.Y.) 350, 957-961, doi:10.1126/science.aad1023 (2015).

27 Scammell, T. E., Arrigoni, E. \& Lipton, J. O. Neural Circuitry of Wakefulness and Sleep. Neuron 93, 747-765, doi:10.1016/j.neuron.2017.01.014 (2017).

28 Weber, F. et al. Regulation of REM and Non-REM Sleep by Periaqueductal GABAergic Neurons. Nature communications 9, 354, doi:10.1038/s41467-017-02765-w (2018).

29 Mochizuki, T. et al. Circadian rhythm of histamine release from the hypothalamus of freely moving rats. Physiology \& behavior 51, 391-394, doi:10.1016/0031-9384(92)90157-w (1992).

30 Inoue, l. et al. Impaired locomotor activity and exploratory behavior in mice lacking histamine H1 receptors. Proceedings of the National Academy of Sciences of the United States of America 93, 1331613320, doi:10.1073/pnas.93.23.13316 (1996).

31 Toyota, H. et al. Behavioral characterization of mice lacking histamine $\mathrm{H}(3)$ receptors. Molecular pharmacology 62, 389-397, doi:10.1124/mol.62.2.389 (2002).

32 Parmentier, R. et al. Anatomical, physiological, and pharmacological characteristics of histidine decarboxylase knock-out mice: evidence for the role of brain histamine in behavioral and sleep-wake control. The Journal of neuroscience : the official journal of the Society for Neuroscience 22, 7695-7711, doi:10.1523/jneurosci.22-17-07695.2002 (2002).

$33 \mathrm{Yu}, \mathrm{X}$. et al. Wakefulness Is Governed by GABA and Histamine Cotransmission. Neuron 87, 164178, doi:10.1016/j.neuron.2015.06.003 (2015).

34 Varin, C., Luppi, P. H. \& Fort, P. Melanin-concentrating hormone-expressing neurons adjust slowwave sleep dynamics to catalyze paradoxical (REM) sleep. Sleep 41, doi:10.1093/sleep/zsy068 (2018).

35 Fabbri, R. et al. Memory retrieval of inhibitory avoidance requires histamine $\mathrm{H} 1$ receptor activation in the hippocampus. Proceedings of the National Academy of Sciences of the United States of America 113, E2714-2720, doi:10.1073/pnas.1604841113 (2016).

36 Nomura, H. et al. Central Histamine Boosts Perirhinal Cortex Activity and Restores Forgotten Object Memories. Biological psychiatry 86, 230-239, doi:10.1016/j.biopsych.2018.11.009 (2019). 
37 Provensi, G., Passani, M. B., Costa, A., Izquierdo, I. \& Blandina, P. Neuronal histamine and the memory of emotionally salient events. British journal of pharmacology $177,557-569$, doi:10.1111/bph.14476 (2020).

$38 \mathrm{Yu}, \mathrm{X}$. et al. Genetic lesioning of histamine neurons increases sleep-wake fragmentation and reveals their contribution to modafinil-induced wakefulness. Sleep 42, doi:10.1093/sleep/zsz031 (2019).

39 Falkner, A. L. et al. Hierarchical Representations of Aggression in a Hypothalamic-Midbrain Circuit. Neuron 106, 637-648.e636, doi:10.1016/j.neuron.2020.02.014 (2020).

40 Lin, D. et al. Functional identification of an aggression locus in the mouse hypothalamus. Nature 470, 221-226, doi:10.1038/nature09736 (2011).

41 Todd, W. D. et al. A hypothalamic circuit for the circadian control of aggression. Nature neuroscience 21, 717-724, doi:10.1038/s41593-018-0126-0 (2018).

42 Michelsen, K. A. et al. Histamine-immunoreactive neurons in the mouse and rat suprachiasmatic nucleus. The European journal of neuroscience 22, 1997-2004, doi:10.1111/j.1460-9568.2005.04387.x (2005).

$43 \mathrm{Kim}, \mathrm{Y}$. S. et al. Histamine resets the circadian clock in the suprachiasmatic nucleus through the H1R-CaV 1.3-RyR pathway in the mouse. The European journal of neuroscience 42, 2467-2477, doi:10.1111/ejn.13030 (2015).

44 Liou, S. Y., Shibata, S., Yamakawa, K. \& Ueki, S. Inhibitory and excitatory effects of histamine on suprachiasmatic neurons in rat hypothalamic slice preparation. Neuroscience letters $41,109-113$, doi:10.1016/0304-3940(83)90231-8 (1983).

45 Stehle, J. Effects of histamine on spontaneous electrical activity of neurons in rat suprachiasmatic nucleus. Neuroscience letters 130, 217-220, doi:10.1016/0304-3940(91)90400-n (1991).

46 Steininger, T. L., Alam, M. N., Gong, H., Szymusiak, R. \& McGinty, D. Sleep-waking discharge of neurons in the posterior lateral hypothalamus of the albino rat. Brain research 840, 138-147, doi:10.1016/s0006-8993(99)01648-0 (1999).

47 Takahashi, K., Lin, J. S. \& Sakai, K. Neuronal activity of histaminergic tuberomammillary neurons during wake-sleep states in the mouse. The Journal of neuroscience : the official journal of the Society for Neuroscience 26, 10292-10298, doi:10.1523/jneurosci.2341-06.2006 (2006).

48 Vanni-Mercier, G., Gigout, S., Debilly, G. \& Lin, J. S. Waking selective neurons in the posterior hypothalamus and their response to histamine $\mathrm{H} 3$-receptor ligands: an electrophysiological study in freely moving cats. Behavioural brain research 144, 227-241, doi:10.1016/s0166-4328(03)00091-3 (2003). 
49 Blandina, P., Munari, L., Provensi, G. \& Passani, M. B. Histamine neurons in the tuberomamillary nucleus: a whole center or distinct subpopulations? Frontiers in systems neuroscience 6, 33, doi:10.3389/fnsys.2012.00033 (2012).

50 Giannoni, P. et al. Heterogeneity of histaminergic neurons in the tuberomammillary nucleus of the rat. The European journal of neuroscience 29, 2363-2374, doi:10.1111/j.1460-9568.2009.06765.x (2009).

51 Umehara, H., Mizuguchi, H. \& Fukui, H. Identification of a histaminergic circuit in the caudal hypothalamus: an evidence for functional heterogeneity of histaminergic neurons. Neurochemistry international 61, 942-947, doi:10.1016/j.neuint.2012.05.022 (2012).

52 Kilkenny, C., Browne, W. J., Cuthill, I. C., Emerson, M. \& Altman, D. G. Improving bioscience research reporting: the ARRIVE guidelines for reporting animal research. PLoS biology 8, e1000412, doi:10.1371/journal.pbio.1000412 (2010).

53 Rogers, A. A. et al. Deep brain stimulation of hypothalamus for narcolepsy-cataplexy in mice. Brain stimulation 13, 1305-1316, doi:10.1016/j.brs.2020.04.006 (2020).

\section{Figures}



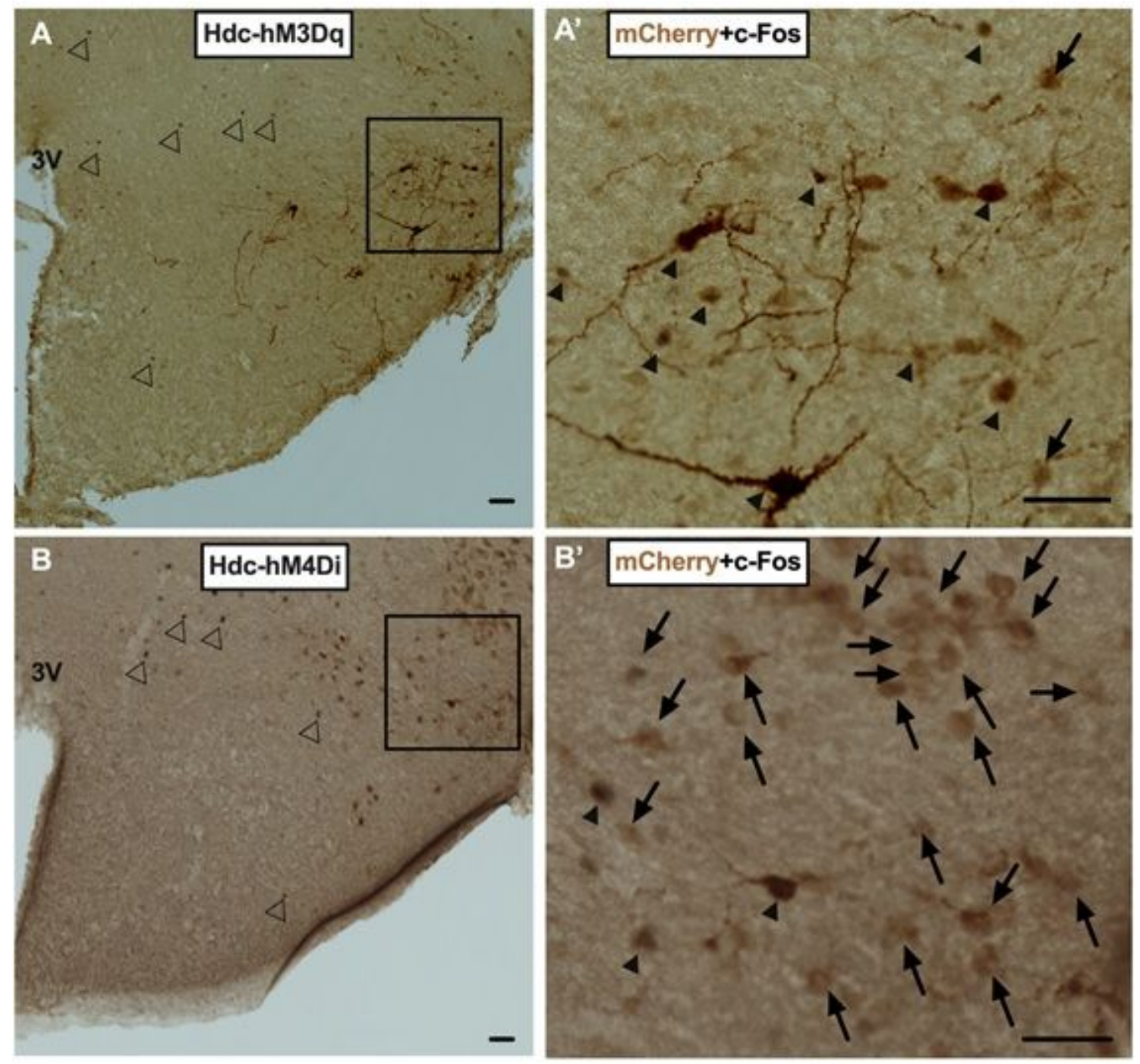

\section{Figure 1}

Specific expression of Hdc-hM3Dq/Hdc-hM4Di in the TMN and confirmation of neuronal activation or inhibition by CNO. A, A representative hypothalamic section from an Hdc-Cre mouse injected with AAVhM3Dq. CNO injection induced c-Fos expression (black nuclei) in mCherry-expressing HATMN neurons (brown) (A'). B, Representative section from an Hdc-Cre mouse injected with AAV-hM4Di. c-Nuclear Fos expression (black) was substantially lower in mCherry-positive neurons (brown) after CNO injection ( $\mathrm{B}^{\prime}$ ). Scale bar $=200 \mu \mathrm{m} .3 \mathrm{~V}$, third ventricle. Arrows: mCherry-positive/c-Fos-negative neurons, Black arrow heads: mCherry-positive/c-Fos-positive neurons, White arrow heads: mCherry-negative/c-Fos-positive neurons. These coronal brain sections were located $-2.18 \mathrm{~mm}$ anterior-posterior from bregma. 


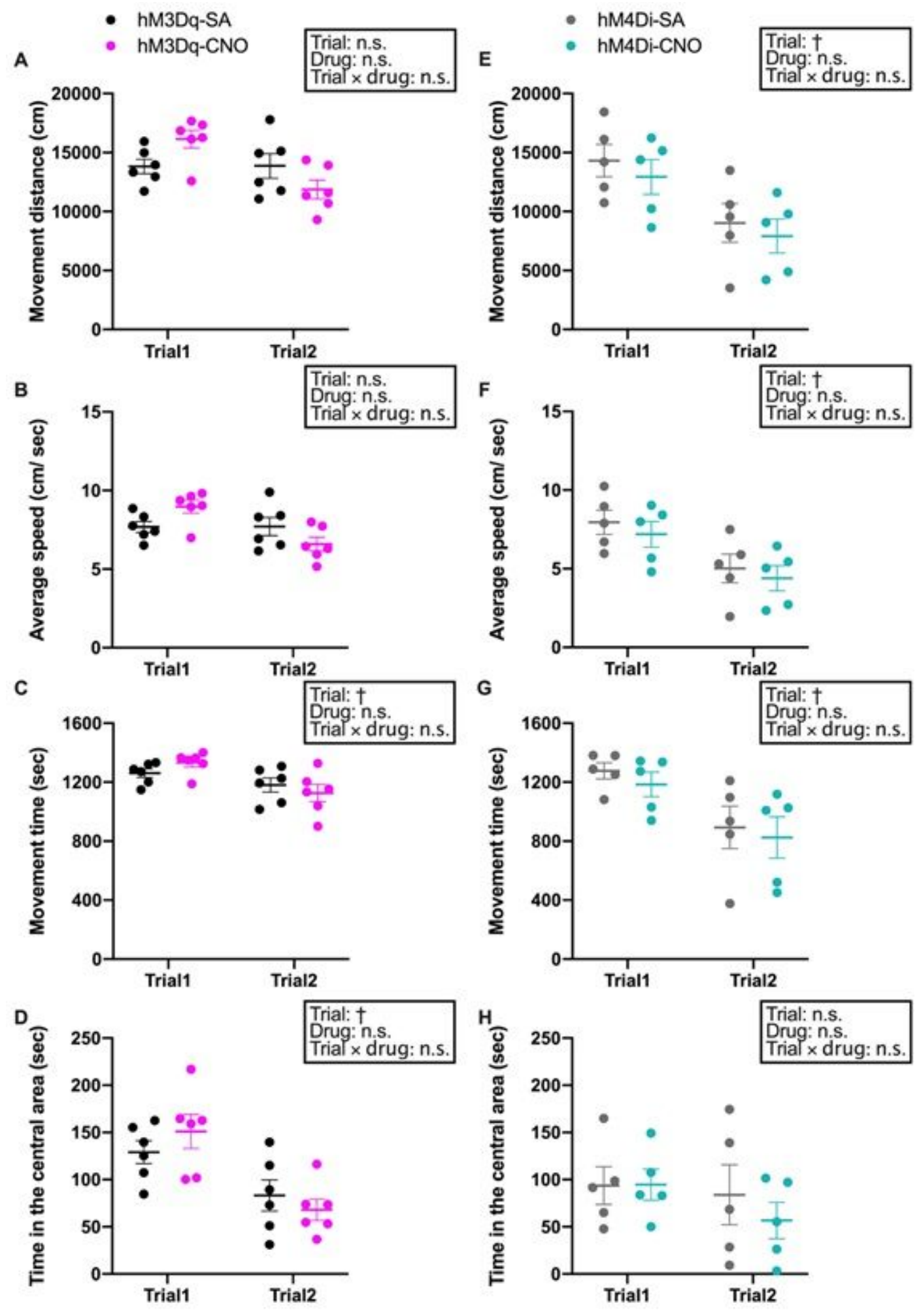

Figure 2

Chemogenetic activation of HATMN neurons enhanced locomotor activity in a novel open field but reduced locomotor activity in a pre-explored (non-novel) open field. Locomotor parameters were compared among Hdc-hM3Dq mice ( $A-D, n=6)$ and Hdc-hM4Di mice $(E-H, n=5)$ for 30 min after CNO or SA injection by two-way RM ANOVA with main factors trial number (1 or 2) and drug treatment (CNO or SA), followed by Sidak's post hoc tests. A, Total movement distance of Hdc-hM3Dq mice after CNO or SA 
injection (trial: $F=4.43, P=0.062$; drug: $F=0.087, P=0.77$; trial $\times$ drug interaction: $F=4.64, P=0.057$ ). $B$, Average locomotion speed of Hdc-hM3Dq mice after CNO or SA injection (trial: $F=4.43, P=0.062$; drug: $F$ $=0.088, P=0.77$; interaction: $F=4.64, P=0.057) . C$, Total movement time of Hdc-hM3Dq mice after CNO or $\mathrm{SA}$ injection (trial: $\mathrm{F}=9.61, \mathrm{P}=0.01$; drug: $\mathrm{F}=0.046, \mathrm{P}=0.83$; interaction: $\mathrm{F}=1.88, \mathrm{P}=0.20$ ). $\mathrm{D}$, Total time spent in the central area by Hdc-hM3Dq mice after CNO or SA injection (trial: $F=21.58, P=0.0009$; drug: $F=0.046, P=0.83$; interaction: $F=1.78, P=0.21$ ). $E$, Total movement distance of Hdc-hM4Di mice after $\mathrm{CNO}$ or $\mathrm{SA}$ injection (trial: $\mathrm{F}=9.44, \mathrm{P}=0.015$; drug: $\mathrm{F}=0.96, \mathrm{P}=0.36$; interaction: $\mathrm{F}=0.0062, \mathrm{P}=$ 0.94). $F$, Average speed of Hdc-hM4Di mice after $C N O$ or $S A$ injection (trial: $F=9.44, P=0.015$; drug: $F=$ 0.96, $P=0.36$; interaction: $F=0.0062, P=0.94)$. G, Total movement time of Hdc-hM4Di mice after CNO or $S A$ injection (trial: $F=8.82, P=0.018$; drug: $F=0.68, P=0.43$; interaction: $F=0.0086, P=0.93$ ). $\mathrm{H}$, Total time spent in the central area by Hdc-hM4Di mice after CNO or SA injection (trial: $F=1.8, P=0.22$; drug: $F$ $=0.24, P=0.64$; interaction: $F=0.63, P=0.45$ ). Data are presented as mean \pm S.E.M. (horizontal bars and error bars) with superimposed individual data points (closed circles). $\uparrow \mathrm{P}<0.05$ by two-way RM ANOVA with main factors 'trial' and 'drug'.

A

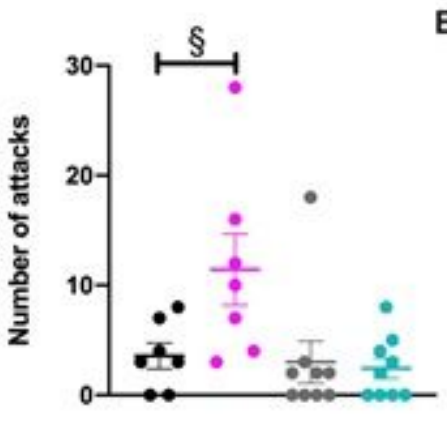

- hM3Dq-SA

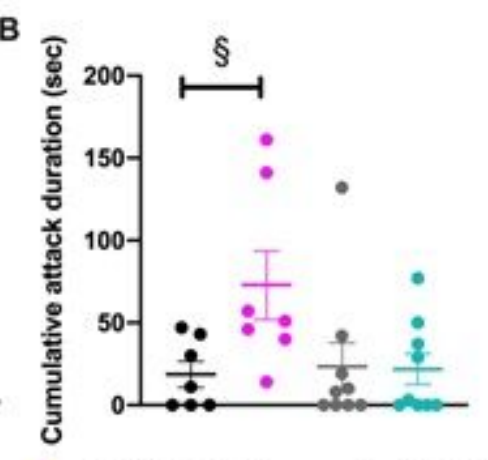

- hM3Dq-CNO
C

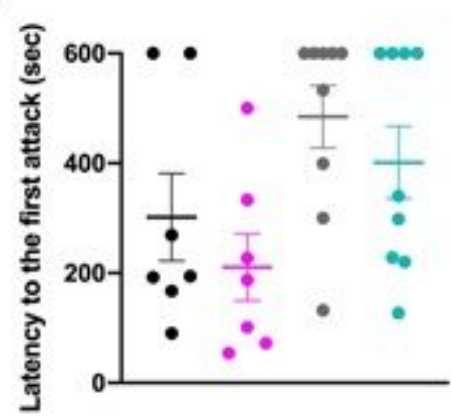

- hM4Di-SA

- hM4Di-CNO

\section{Figure 3}

Chemogenetic activation of HATMN neurons enhanced aggression in the resident-intruder test. A, Number of aggressive behaviours in 10 min by Hdc-hM3Dq and Hdc-hM4Di mice after CNO or SA injection ( $n=7$ or $9 ; P=0.035$ by Mann-Whitney $U$ test). $B$, Cumulative duration of aggressive behaviours within 10 min by Hdc-hM3Dq and Hdc-hM4Di mice after CNO or SA injection ( $\mathrm{n}=7$ or 9, $\mathrm{P}=0.018$ by Mann-Whitney U test). C, Latency to first aggressive behaviour by Hdc-hM3Dq and Hdc-hM4Di mice after CNO or SA injection ( $n=7$ or 9; no significant differences by Mann-Whitney $U$ test). Data are presented as mean \pm S.E.M. (horizontal bars and error bars) with superimposed individual data points (closed circles). § $\mathrm{P}<0.05$. 

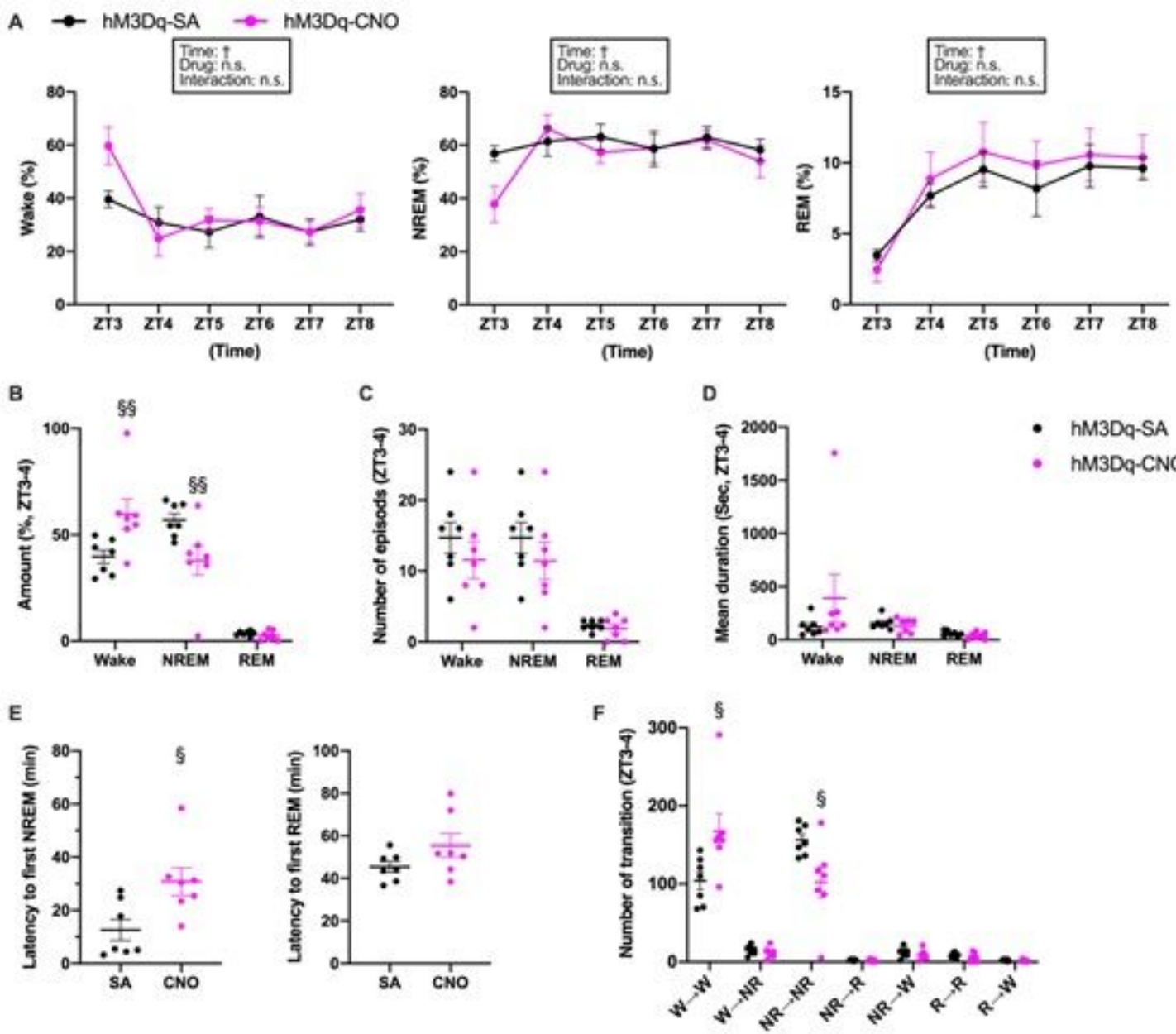

\section{Figure 4}

Chemogenetic activation of HATMN neurons increased wakefulness and decreased NREM sleep during light periods. Hdc-hM3Dq mice $(n=7)$ were injected with CNO or SA at the indicated times and wake and sleep phases monitored by EEG/EMG. A, Relative mean hourly wake, NREM sleep and REM sleep times (\%) after CNO or SA injection at ZT3 (wake - time: $F=4.34, P=0.0019$; drug factor: $F=1.24, P=0.29$; time $\times$ drug interaction: $F=1.28, P=0.29 ;$ NREM - time: $F=3.06, P=0.016$; drug: $F=1.31, P=0.28$; interaction: $F=1.46, P=0.22$; REM - time: $F=8.21, P<0.0001$; drug: $F=0.44, P=0.52$; interaction: $F=$ $0.23, \mathrm{P}=0.95$; ; all by two-way RM ANOVA with main factors time and drug, followed by Sidak's post hoc test). B, Mean wake, NREM sleep and REM sleep times during ZT3-4 after CNO or SA injection at ZT3 (wake $P=0.0070$ and NREM $P=0.0087$ by Mann-Whitney $U$ test). C, Number of wake or sleep phase episodes during ZT3-4 after CNO or SA injection at ZT3 (no significant differences by Mann-Whitney U test). D, Mean duration of sleep-wake status after CNO or SA injection at ZT3 (no significant differences by Mann-Whitney U test). E, Latency to the first NREM and REM sleep episode after CNO or SA injection at ZT3 (NREM P $=0.018$ by Mann-Whitney $U$ test). F, Frequencies of specific sleep-wake status transitions after CNO or SA injection at ZT3 (wake " wake $P=0.0064$, wake " NREM $P=0.30$, NREM " NREM $P=0.018$, NREM " REM $P=0.69$, NREM " wake $P=0.36$, REM " REM $P=0.30$ and REM " wake $P=$ 0.32 by Mann-Whitney $U$ test). Data are presented as mean \pm S.E.M. (horizontal bars and error bars) of $n$ $=7$ mice with $(B-F)$ or without $(A)$ superimposed individual data points (close circles). $+P<0.05$ by two- 
way RM ANOVA with main factors 'time' and 'drug'; * $P<0.05$ by post hoc Sidak's multiple comparisons test; $\S \mathrm{P}<0.05$, $\S \mathrm{P}<0.01$ by Mann-Whitney $\mathrm{U}$ test.
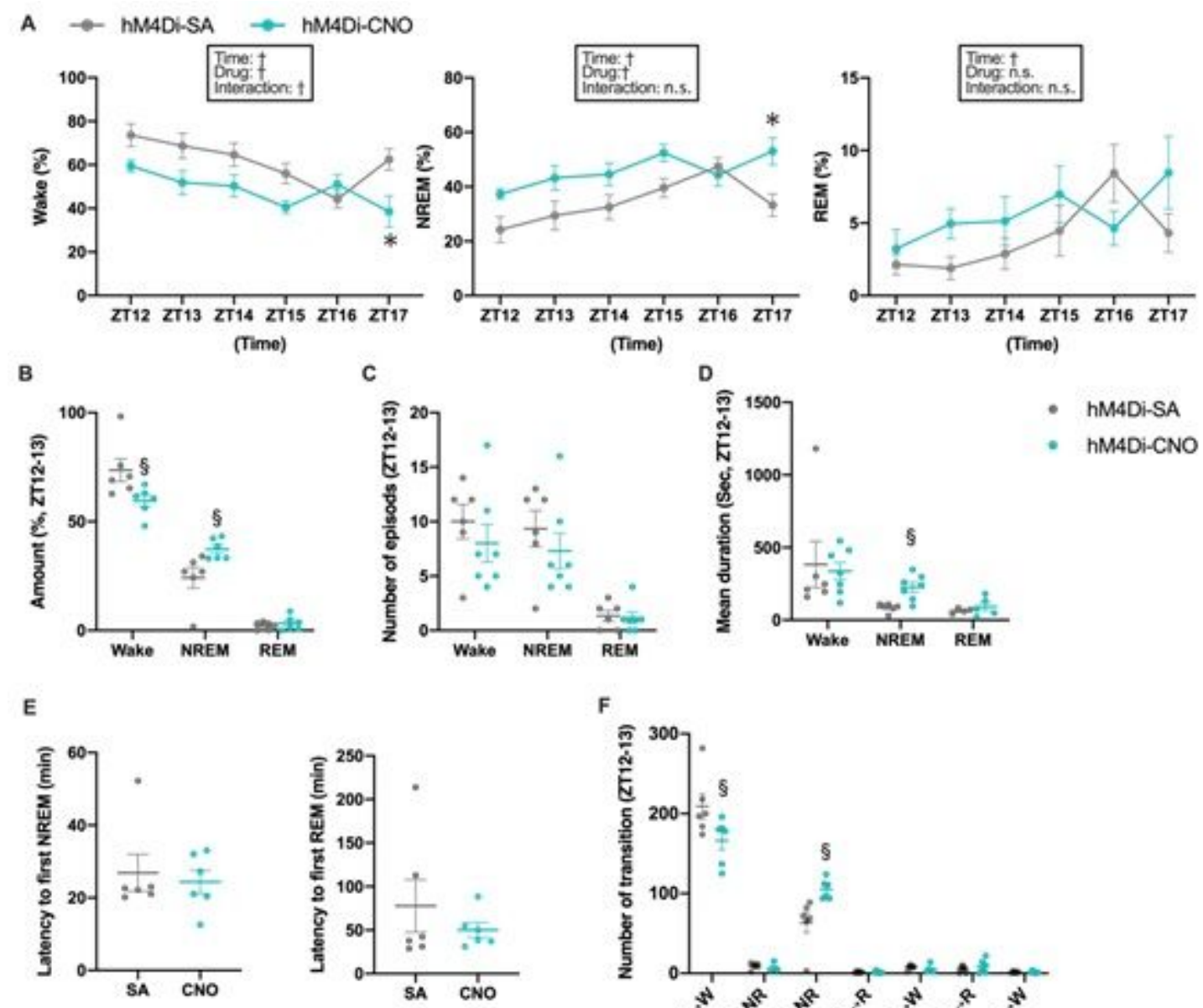

- hM4Di-CNO
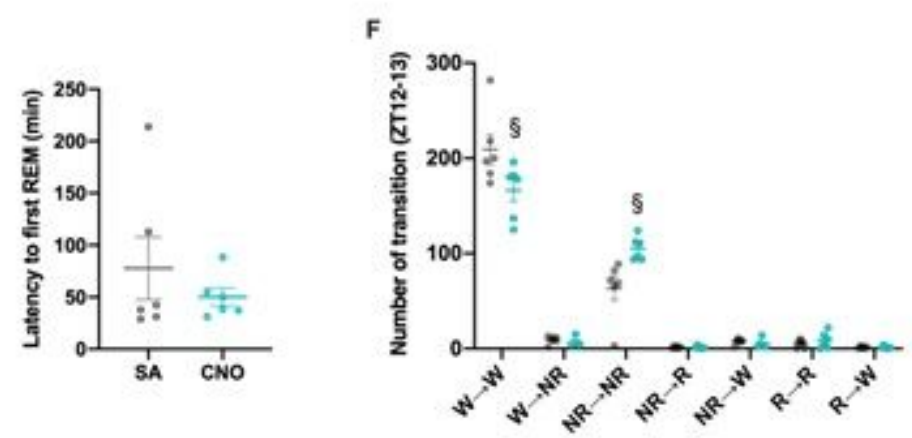

Figure 5

Chemogenetic inhibition of HATMN neurons decreased wakefulness and increased NREM sleep during dark periods. Hdc-hM3Di mice $(n=6)$ were injected with CNO or SA at the indicated time and wake and sleep phases monitored by EEG/EMG. A, Hourly mean wake, NREM sleep and REM sleep durations after CNO or SA injection at ZT12 (wake - time: $F=5.99, P=0.0002$; drug: $F=10.02, P=0.010$; time $\times$ drug interaction: $F=2.81, P=0.026$; NREM - time: $F=5.65, P=0.0003$; drug: $F=10.39, P=0.0091$; interaction: $F=2.28, P=0.061$; REM - time: $F=3.18, P<0.014$; drug: $F=1.23, P=0.29$; interaction: $F=$ $2.32, \mathrm{P}=0.057$; all analyses by two-way RM ANOVA with main factors time and drug, followed by Sidak's post hoc test). B, Mean wake, NREM sleep and REM sleep durations during ZT12-13 after CNO or SA injection at ZT12 (wake $P=0.015$ and NREM $P=0.013$ by Mann-Whitney $U$ test) $C$, Number of episodes during ZT12-13 after CNO or SA injection at ZT12 (no significant differences by Mann-Whitney U test). D, Mean duration of sleep and wake stages after CNO or SA injection at ZT12 (no significant differences by Mann-Whitney $U$ test). E, Latency to the first NREM and REM sleep episodes after CNO or SA injection at ZT12 (no significant difference by Mann-Whitney U test). F, Sleep-wake status transitions after CNO or SA injection at ZT12 (wake " wake $P=0.041$, wake " NREM $P=0.29$, NREM " NREM $P=0.0022$, NREM " REM $P=0.93$, NREM " wake $P=0.22$, REM " REM $P=0.51$ and REM " wake $P=0.93$ by Mann-Whitney $U$ 
test). Data are presented as mean \pm S.E.M. of $n=6$ mice with $(B-F)$ or without $(A)$ superimposed individual data points (closed circles). $+\mathrm{P}<0.05$ by two-way RM ANOVA with main factors 'time' and 'drug', * $\mathrm{P}<0.05$ by post hoc Sidak's multiple comparisons test. $\S \mathrm{P}<0.05$ by Mann-Whitney $\mathrm{U}$ test.

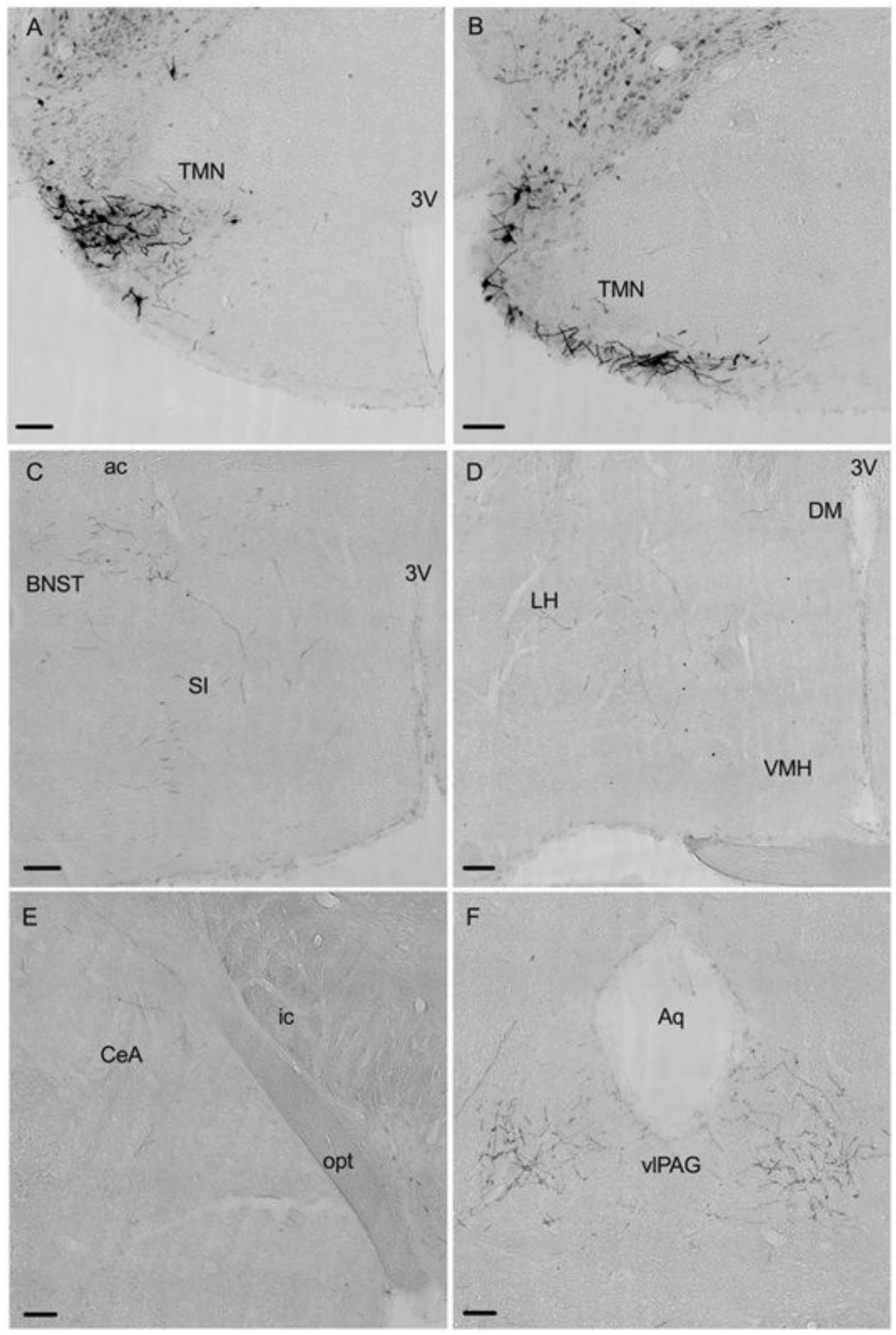

Figure 6

Projection targets of HATMN neurons. The presence of mCherry-immunoreactive HATMN somata and fibres were examined in coronal brain sections 6 months after AAV injections. A, mCherry-immunoreactive 
HATMN neuronal somata and fibres in the ventrolateral TMN. B, mCherry-immunoreactive HATMN neuronal somata and fibres in the caudal TMN. C-F, HATMN fibres in the bed nucleus of stria terminalis (BNST) (C), lateral hypothalamus (LH) (D), central amygdala (CeA) (E) and ventrolateral periaqueductal grey (vIPAG) (F). Scale bar $=100 \mu \mathrm{m}$. ac, anterior commissure; Aq, aqueduct; DM, dorsomedial hypothalamic nucleus; LA, lateroanterior hypothalamic nucleus; MPO, medial preoptic nucleus; opt, optic tract; SI, substantia innominata; $\mathrm{VMH}$, ventromedial hypothalamic nucleus;3V, third ventricle. Anteriorposterior locations ( $\mathrm{mm}$ from bregma): (A) $-2.18 \mathrm{~mm}$, (B) $-2.70 \mathrm{~mm}$, (C) $0.14 \mathrm{~mm}$, (D) $-0.46 \mathrm{~mm}$, (E) $-1.34 \mathrm{~mm},(\mathrm{~F})-4.24 \mathrm{~mm}$.

\section{Supplementary Files}

This is a list of supplementary files associated with this preprint. Click to download.

- HdcCreSupplsubmitted.docx 